\title{
Quantum tunnelling in the deformed region of the LMG model
}

\author{
A Vourdas and R F Bishop \\ Department of Mathematics, UMIST, PO Box 88, Manchester M60 1QD, UK
}

Received 11 June 1984, in final form 18 July 1984

\begin{abstract}
We present classical solutions that describe quantum-mechanical tunnelling between the two minima in the deformed region $(\lambda>1)$ of the LMG model.
\end{abstract}

In view of the complexity of realistic calculations for quantum many-body systems, approaches using model Hamiltonians as their starting point have considerable interest for the application of either new many-body methods or new approximation schemes. In order to be of more than just academic interest, such model Hamiltonians need, however, to mirror the main properties of the particular real system, and yet be simple enough to be tractable. In this context the model of Lipkin et al (1965a, b, c; hereafter referred to as the LMG model) (and see Agassi et al 1966) is an interesting prototype of the nuclear shell model, which has been much studied in the literature by a wide variety of theoretical techniques. The model was first introduced in connection with the collective monopole vibrations (or breather modes) for spherical nuclei, although it has also been indicated (Lührmann 1977) that, below the critical region, the model has ground-state correlations that resemble those in a system with long-range correlations, such as the electron gas. For the present purposes it is also important to realise that, although the LMG model is undoubtedly too idealised to be of any real quantitative use for nuclei, it serves as a stepping stone to other more complicated but more realistic Hamiltonians which may similarly be constructed from the generators of the Lie algebras of higher groups. The interacting boson model (IBM) of Arima and Iachello (1976) is a particular example of relevance here.

In the present context we will be interested in applying functional integral techniques and semiclassical approximations to the LMG model. Functional integrals and the related techniques involving coherent states have recently begun to be used extensively in the study of the nuclear many-body problem (see, e.g., Blaizot and Orland 1981, Koonin 1982, Negele 1982). Functional integrals for the spin coherent states are needed for application to the LMG model. Spin coherent states have been studied by Arecchi et al (1972) and Perelomov $(1972,1977)$ and, more recently, the application of functional integration techniques to them has been performed by Klauder (1979) and Jevicki and Papanicolaou (1979). Preliminary applications of these techniques to the study of the LMG model have recently been discussed by Gilmore and Feng (1978), Kan et al (1979, 1980), Levit et al (1980a), Kan (1980), Shankar (1980) and Koonin (1982). A particularly interesting new approach to the LMG model has also recently been given by Arponen (1982), in which the 
usual coupled-cluster (or $\exp (S)$ ) formulation of quantum many-body theory has been extended by exploiting the stationary property of a particular energy functional with respect to two distinct types of linked-cluster amplitudes. This formalism is also particularly helpful in so far as it bears a very close resemblance both to the methods above based on coherent states and to the mean-field theory or semiclassical approximation (see e.g., Klein and Li 1981).

We start with the Hamiltonian for the LMG model in its original form as

$$
H=\frac{1}{2} \varepsilon \sum_{n=1}^{N} \sum_{\sigma= \pm 1} \sigma a_{n \sigma}^{\dagger} a_{n \sigma}+\frac{1}{2} V \sum_{n, n^{\prime}=1}^{N} \sum_{\sigma= \pm 1} a_{n \sigma}^{\dagger} a_{n^{\prime} \sigma}^{+} a_{n^{\prime}-\sigma} a_{n-\sigma}
$$

where the operators $a_{n \sigma}^{\dagger}$ are the nucleon creation operators for the only two levels, $\sigma= \pm 1$, contained in the model and $N$ is the total number of particles. The Hamiltonian (1) can be transformed to a pseudospin representation in the usual way by means of the identifications

$$
\begin{array}{ll}
J_{z} & =\frac{1}{2} \sum_{n, \sigma} \sigma a_{n \sigma}^{\dagger} a_{n \sigma} \\
J_{+} & =\sum_{n} a_{n,+1}^{\dagger} a_{n,-1}
\end{array}
$$

where the pseudospin operators $\left(J_{+}, J_{-}, J_{z}\right)$ are the usual SU(2) generators. The resulting pseudospin Hamiltonian

$$
H=\varepsilon J_{z}+\frac{1}{2} V\left(J_{+}^{2}+J_{-}^{2}\right)
$$

may then be diagonalised in each fixed- $J$ SU(2)-invariant subspace (of dimension $2 J+1$ ) since the Casimir operator $J^{2}$ formed from the pseudospin operators (2) commutes with the Hamiltonian (3). We shall consider only the subspace which contains the ground state and which therefore has the maximum possible value of $J$, namely $J=\frac{1}{2} N$, since the 'unperturbed' $(V=0)$ ground state has $J_{z}=-\frac{1}{2} N$. In terms of the familiar SU(2) Euler angles $(\theta, \varphi) \equiv \Omega$, the spin coherent state (or atomic coherent state) $|\Omega\rangle$ may be obtained by applying a particular unitary transformation $\mathrm{U}(\Omega)$ to the state $\left|J, J_{z}=-J\right\rangle$ in the fixed- $J$ subspace. The unitary transformations $U(\Omega)$ and the spin coherent states $|\Omega\rangle$ are then in a one-to-one correspondence with points on the surface of a sphere $(0 \leqslant \theta \leqslant \pi ; 0 \leqslant \varphi \leqslant 2 \pi)$, which in the analogous case of quantum optics is referred to as the Bloch sphere.

Lieb (1973) used the spin coherent states $|\Omega\rangle$ to define the quantity $H_{Q}$ as the expectation value of the Hamiltonian in the spin coherent state representation and evaluated it as

$$
\begin{aligned}
H_{Q} & \equiv\langle\Omega|H| \Omega\rangle \\
& =-\frac{1}{2} N \varepsilon\left(\cos \theta+\frac{1}{2} \lambda \sin ^{2} \theta \cos 2 \varphi\right)
\end{aligned}
$$

where

$$
\lambda \equiv(N-1) V / \varepsilon
$$

The Bogoliubov inequality $\langle\psi|\exp (\hat{O})| \psi\rangle \geqslant \exp \langle\psi|\hat{O}| \psi\rangle$ for arbitrary operator $\hat{O}$ and state 
$|\psi\rangle$ may then be used to find a lower bound for the quantum partition function

$$
\begin{aligned}
\operatorname{Tr}(\exp (-\beta H)) & =\frac{(2 J+1)}{4 \pi} \int \mathrm{d} \Omega\langle\Omega|\exp (-\beta H)| \Omega\rangle \\
& \geqslant \frac{(2 J+1)}{4 \pi} \int \mathrm{d} \Omega \exp (-\beta\langle\Omega|H| \Omega\rangle) \\
& =\frac{(2 J+1)}{4 \pi} \int \mathrm{d} \Omega \exp \left(-\beta H_{\mathrm{Q}}\right) .
\end{aligned}
$$

Taking the zero-temperature limit $(\beta \rightarrow \infty)$, one finds that $\min \left\{H_{\mathrm{Q}}\right\}$ is an upper bound for the ground-state energy. Indeed, the quantity $\min \left\{H_{\mathrm{Q}}\right\}$ provides a very good approximation for the real ground-state energy for large $N$ (see, e.g., Gilmore and Feng 1978).

Authors who work within the alternative mean-field theory or who use the language of time-dependent Hartree-Fock (TDHF) theory use states equivalent to these coherent states and arrive at a Hartree-Fock energy identical with the quantity $H_{\mathrm{Q}}$ of equation (4). That the two languages and formalisms are fully equivalent has been explained particularly clearly by Kan et al (1980). Kan et al (1979) also show how the general TDHF equation can be written in Hamilton's canonical form. For the present LMG model $\varphi$ and $\cos \theta$ are shown to play the roles of canonical coordinate and momentum, respectively. In particular, introduction of the canonically conjugate variables

$$
q \equiv \varphi \quad p \equiv-\frac{1}{2} N \hbar \cos \theta
$$

leads to the classical equations of motion from the semiclassical Hamiltonian $H_{\mathrm{Q}}$ of equation (4) (or, equivalently, the TDHF equations) taking the canonical form

$$
\mathrm{d} p / \mathrm{d} t=-\partial H_{\mathrm{Q}} / \partial q \quad \mathrm{~d} q / \mathrm{d} t=\partial H_{\mathrm{Q}} / \partial p
$$

and hence

$$
\begin{aligned}
& \hbar \mathrm{d}(\cos \theta) / \mathrm{d} t=\lambda \varepsilon\left(1-\cos ^{2} \theta\right) \sin 2 \varphi \\
& \hbar \mathrm{d} \varphi / \mathrm{d} t=\varepsilon(1-\lambda \cos \theta \cos 2 \varphi) .
\end{aligned}
$$

A trivial minimisation of $H_{\mathrm{Q}}$ from equation (4) shows that, for $\lambda<1$, the state with minimum energy lies at the north pole $(\theta=0)$ of the Bloch sphere. Conversely, for $\lambda>1$, the minimum energy occurs at the two points $A$ and $B$ on the Bloch sphere specified in terms of their polar coordinates $(\theta, \varphi)$ as $\left(\cos ^{-1} \lambda^{-1}, 0\right)$ and $\left(\cos ^{-1} \lambda^{-1}, \pi\right)$, respectively. The minimal value of the energy at both these points is

$$
E_{\mathrm{A}}=E_{\mathrm{B}}=-\frac{1}{4} N \varepsilon\left(\lambda+\lambda^{-1}\right)
$$

In this case $(\lambda>1)$ the north pole is now a saddle point of the energy functional (4). We might also mention that, similarly, the state of maximum energy from equation (4) lies at the south pole $(\theta=\pi)$ for $\lambda<1$ and at the two points $\mathrm{A}^{\prime}\left(\cos ^{-1}\left(-\lambda^{-1}\right), \frac{1}{2} \pi\right)$ and $\mathrm{B}^{\prime}\left(\cos ^{-1}\left(-\lambda^{-1}\right), \frac{3}{2} \pi\right)$ in the southern hemisphere for $\lambda>1$.

The qualitative change that occurs at the point $\lambda=1$ in this mean-field or coherent-state approximation to the exact LMG model is usually referred to as the phase transition from the spherical $(\lambda<1)$ to the deformed $(\lambda>1)$ shell. It has long been known that the exact model undergoes a similar phase transition into a deformed phase as the interaction coupling constant $V$ (or, equivalently, $\lambda$ ) increases beyond some critical value (Agassi et al 
1966). An important feature missing from the essentially classical mean-field or coherentstate approach presented so far is the possibility of quantum tunnelling in the case $\lambda>1$ between the two degenerate energy minima A and B, separated by a potential barrier. This twofold degeneracy has already been discussed in the original formulation of the model by Agassi et al (1966) by using the projection method. In general, whenever a Hartree-Fock solution breaks a symmetry of the original Hamiltonian, an associated degeneracy of that Hartree-Fock solution is expected, and itself is generally associated with a set of low-lying collective excitations that are nearly degenerate with the ground state. The degenerate Hartree-Fock solutions are then linear combinations of these collective states, whose spectrum may be obtained from the Hartree-Fock solution by projecting out states of definite symmetry. A well known example is the case of deformed nuclei where breaking of rotational invariance leads to a degenerate (infinite) set of Hartree-Fock states with respect to the orientation of the deformed states. In this case, projecting out states of good angular momentum gives a set of states which, if the approximation is valid, should comprise the members of the rotational band based on the ground state. In the present case of the LMG model, the broken symmetry corresponds not to a continuous transformation but to a twofold degeneracy in the Hartree-Fock solutions arising from the symmetry under $\varphi \rightarrow \varphi+\pi$ of equation (4). The two Hartree-Fock solutions arising from this degeneracy are transformed one into the other by the parity transformation, and states of definite parity are readily obtained by taking the sum and difference of the degenerate Hartree-Fock states. More recently the parity-projection technique has been applied to the LMG model by Kan (1980).

Returning to the functional integral approach to the LMG model, it has been suggested that quantum tunnelling should be discussed with the standard semiclassical techniques. In order to do this we need both (i) to find a classical solution which describes the quantum tunnelling and then (ii) to use this solution in semiclassical approximations to the functional integral. In the remainder of this work, we address only the first of these points.

A very convenient method of applying classical techniques to study a classically forbidden, but quantum mechanically penetrable, region is to permit one or more of the real physical variables (e.g., time or momentum) to acquire complex values. We can see that this procedure is reasonable if we recall that in the classically allowed regions one has asymptotically free plane-wave states proportional to $\exp (\mathrm{ipx})$, whereas in the classically forbidden region the quantum-mechanical bound state becomes asymptotically proportional to $\exp (-p x) \equiv \exp [\mathrm{i}(\mathrm{i} p) x]$. This technique of introducing complex coordinates was introduced by Keller (1962) in the context of a geometrical theory of diffraction. If some of the relevant variables are analytically continued in this manner from the real line into the complex plane, the geometrical optics (cf classical mechanics) can then offer a very good approximation for the wave optics (cf quantum mechanics). Geometrical optics in the real domain clearly fail completely to describe the 'geometrically shadowed' regions, and it is just this restriction that is overcome by the technique of analytic continuation. Which particular physical variable should be chosen to be analytically continued depends very much on the problem under discussion. The technique has been applied widely to various problems in mathematical physics (see, e.g., Keller 1962, McLaughlin 1972, Miller and George 1972, Balian and Bloch 1974, Koeling and Malfeit 1975, Knoll and Schaeffer 1976, Schulman 1981). In particle physics the technique led to the introduction of instantons (see, e.g., Coleman 1979), and in nuclear physics the technique was recently applied by Levit et al (1980b) to fission problems.

For our particular problem we will see that it is appropriate to extend the real time $t$ to imaginary values $(t \rightarrow \mathrm{i} \tau)$, and to allow the canonical momentum variable $p$ of equation (7) 
to take complex values. The starting point is equation (4) for the 'classical' energy, $H_{\mathrm{Q}}$. When $H_{\mathrm{Q}}$ achieves the minimum energy of equation (10) in the case of interest $(\lambda>1)$, equation (4) reduces to

$$
\lambda+\lambda^{-1}-2 \cos \theta-\lambda \sin ^{2} \theta \cos 2 \varphi=0
$$

which is readily solved for $\cos \theta$ as

$$
\cos \theta=\frac{1 \pm\left[\left(1-\lambda^{2} \cos 2 \varphi\right)(1-\cos 2 \varphi)\right]^{1 / 2}}{\lambda \cos 2 \varphi}
$$

If this value for $\cos \theta$ is now substituted into equation $(9 b)$ for the time evolution of $\varphi$ we get

$$
\hbar \mathrm{d} \varphi / \mathrm{d} t=\mp \varepsilon\left[\left(1-\lambda^{2} \cos 2 \varphi\right)(1-\cos 2 \varphi)\right]^{1 / 2} .
$$

In order now to study the quantum tunnelling between the two degenerate energy minima (10) in this case $(\lambda>1)$, we analytically continue equation (13) onto the imaginary time axis, $t \rightarrow \mathrm{i} \tau$, to get

$$
\hbar \mathrm{d} \varphi / \mathrm{d} \tau=\mp \varepsilon\left[\left(\lambda^{2} \cos 2 \varphi-1\right)(1-\cos 2 \varphi)\right]^{1 / 2} .
$$

Straightforward integration of equation (14) leads to the result

$$
\begin{aligned}
& \sin ^{2} \varphi=\left(1-\tanh ^{2} \tau^{\prime}\right)\left(\frac{2 \lambda^{2}}{\lambda^{2}-1}-\tanh ^{2} \tau^{\prime}\right)^{-1} \\
& \tau^{\prime} \equiv \pm \varepsilon\left(2 \lambda^{2}-2\right)^{1 / 2} \tau / \hbar+C
\end{aligned}
$$

where $C$ is an arbitrary constant which simply defines the origin of the imaginary time coordinate $\tau$. The right-hand side of expression (15) is readily checked to lie between zero and one, as it should do for real $\varphi$ given by the left-hand side. It is also readily verified that the solution (15) satisfies the condition $\lambda^{2} \cos 2 \varphi-1>0$ as required in equation (14). Finally, substituting equation (15) into equation (12) gives the corresponding solution for $\cos \theta$ :

$$
\begin{gathered}
\cos \theta=\frac{1}{\lambda} \frac{2 \lambda^{2}-\left(\lambda^{2}-1\right) \tanh ^{2} \tau^{\prime}}{2+\left(\lambda^{2}-1\right) \tanh ^{2} \tau^{\prime}} \pm \mathrm{i} \frac{\left(\lambda^{2}-1\right)\left(2 \lambda^{2}+2\right)^{1 / 2}}{\lambda} \frac{\tanh \tau^{\prime} \operatorname{sech} \tau^{\prime}}{2+\left(\lambda^{2}-1\right) \tanh ^{2} \tau^{\prime}} \\
\tau^{\prime} \equiv \pm \varepsilon\left(2 \lambda^{2}-2\right)^{1 / 2} \tau / \hbar+C .
\end{gathered}
$$

From equations (15) and (16) it is readily seen that in the asymptotic limit $\tau \rightarrow \pm \infty, \varphi \rightarrow 0, \pi$ and $\cos \theta \rightarrow \lambda^{-1}$, and hence in this limit we regain the two classically stable, degenerate minima $\mathbf{A}$ and $\mathbf{B}$. Conversely, for finite values of $\tau$, the solution enters the classically forbidden region and the canonical momentum $p$, proportional to $\cos \theta$, acquires complex values as required.

From the solution so obtained, we may also calculate the analytically continued classical action, namely

$$
S=\int_{-\infty}^{\infty}\left(p \frac{\mathrm{d} q}{\mathrm{~d} \tau}-H\right) \mathrm{d} \tau,
$$

by substituting for the canonical variables $p$ and $q$ from equation (7) and for the Hamiltonian from equation (10). Using the solution of equations (15) and (16), the result is then finite provided that, as expected, we redefine the minimum energy of equation (10) to 
be the new zero level. After a lengthy integration we obtain

$$
\begin{aligned}
S_{0} & \equiv \int_{-\infty}^{\infty} p \frac{\mathrm{d} q}{\mathrm{~d} \tau} \mathrm{d} \tau \\
& =N \hbar\left(\lambda^{-1} \tan ^{-1}\left[\frac{1}{2}\left(\lambda^{2}-1\right)\right]^{1 / 2}-\tanh ^{-1}\left[\left(\lambda^{2}-1\right) / 2 \lambda^{2}\right]^{1 / 2}\right)
\end{aligned}
$$

As we have already mentioned, the possibility of quantum tunnelling splits the two degenerate minima $E_{\mathrm{A}}=E_{\mathrm{B}} \equiv E_{0}$ of equation (10) symmetrically in this case $(\lambda>1)$ into the levels $E_{0} \pm \Delta E$. In the familiar WKB approximation (see, e.g., Coleman 1979), the splitting is given simply as

$$
\Delta E \propto \exp \left(-S_{0}\right)
$$

with $S_{0}$ given by equation (18). Well known limitations of the wKB approximation mean that the pre-factor in equation (19) is only approximate, although the exponential factor, which for many purposes is the most important, is given accurately.

Calculation of the determinant which takes gaussian fluctuations around our semiclassical solution into account is necessary for the first correction to (the pre-factor of) the result (19). Also, further more refined functional integral calculations may be performed to give corrections of higher order in $\hbar$ which may be regarded as quantum fluctuations around our 'semiclassical path' to tunnelling. These further calculations are by no means trivial, but it is our belief that there is enough experience available from the instanton problem to assist in further analysis of the present LMG model.

What is important for this first calculation is that we have succeeded in lifting explicitly the 'classical degeneracy' of the LMG model for the case $\lambda>1$ using the 'instanton-like' solution that we have obtained analytically.

\section{Acknowledgment}

We gratefully acknowledge support for this work in the form of a Research Grant from the Science and Engineering Research Council of Great Britain.

\section{References}

Agassi D, Lipkin H J and Meshkov N 1966 Nucl. Phys. 86321

Arecchi F T, Courtens E, Gilmore R and Thomas H 1972 Phys, Rev. A 62211

Arima A and Iachello F 1976 Ann. Phys., NY 99253

Arponen J 1982 J. Phys. G: Nucl. Phys. 8 L129

Balian R and Bloch C 1974 Ann. Phys., NY 85514

Blaizot J P and Orland H 1981 Phys. Rev. C 241740

Coleman S 1979 The Whys of Subnuclear Physics (Proc. Int. School of Subnuclear Physics, Erice, 1977) ed. A Zichichi (New York: Plenum)

Gilmore R and Feng D H 1978 Phys. Lett. 76B 26

Jevicki A and Papanicolaou N 1979 Ann. Phys., NY 120107

Kan K K 1980 Phys. Rev. C 222228

Kan K K, Griffin J J, Lichtner P C and Dworzecka M 1979 Nucl. Phys. A 332109

Kan K K, Lichtner P C, Dworzecka M and Griffin J 1980 Phys. Rev. C 211098

Keller J B 1962 J. Opt. Soc. Am. 52116

Klauder J 1979 Phys. Rev. D 192349

Klein A and Li C T 1981 Phys. Rev. Lett. 46895 
Knoll J and Schaeffer R 1976 Ann. Phys., NY 97307

Koeling $T$ and Malfiet R A 1975 Phys. Rep. 22181

Koonin S E 1982 Nuclear Theory 1981 ed. G F Bertsch (Singapore: World Scientific) p 184 Levit S, Negele J W and Paltiel Z 1980a Phys. Rev. C 211603

- 1980b Phys. Rev. C 221979

Lieb E H 1973 Commun. Math. Phys. 31327

Lipkin H J, Meshkov N and Glick A J 1965a Nucl. Phys. 62188

- 1965b Nucl. Phys. 62199

1965c Nucl. Phys. 62211

Lührmann K H 1977 Ann. Phys., NY 103253

McLaughlin D W 1972 J. Math. Phys. 131099

Miller W H and George T F 1972 J. Chem. Phys. 565668

Negele J W 1982 Rev. Mod. Phys. 54913

Perelomov A M 1972 Commun. Math. Phys. 26222

—_ 1977 Sov. Phys.-Usp. 20703

Schulman L S 1981 Techniques and Applications of Path Integration (New York: Wiley)

Shankar R 1980 Phys. Rev. Lett. 451088 